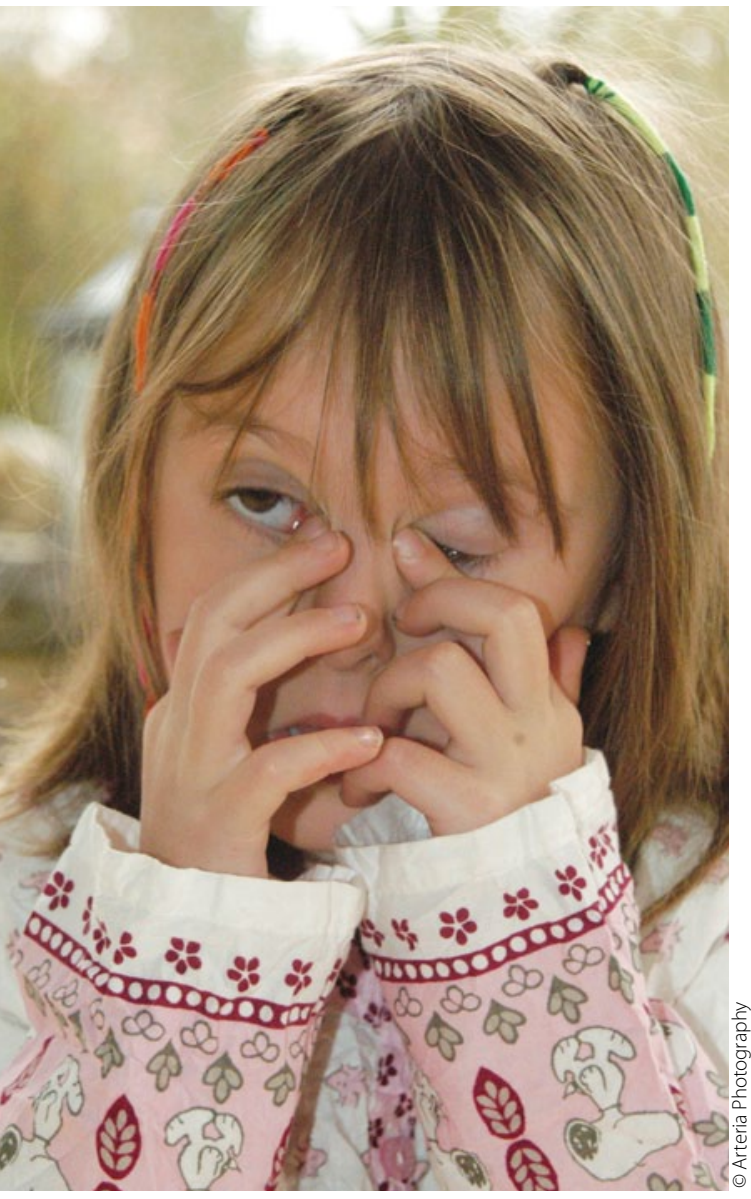

Allergische Rhinitis: Weder die Schulnoch die Alternativmedizin haben eine überzeugende Lösung.

\section{Akupunktur führt zwar zu einer statistisch signifikanten Besserung der Symptome einer allergischen Rhinitis, die aber so gering ausfällt, dass der praktische Nutzen fraglich erscheint.}

— Ein Viertel bis die Hälfte von Personen mit allergischer Rhinitis sucht im Lauf des Lebens unter anderem auch Hilfe bei alternativmedizinischen Verfahren. Man kann diesen hohen Prozentsatz als Indiz dafür werten, dass die schulmedizinischen Methoden auch nicht gerade das Gelbe vom Ei sind.

\title{
Akupunktur und Wissenschaft: eine unglückliche Beziehung
}

422 Personen mit IgE-positiver allergischer Rhinitis auf Birken- und Gräserpollen wurden verblindet in drei Gruppen randomisiert. 212 Personen erhielten von in der Methode erfahrenen Ärzten im Lauf von zwei Monaten zwölf Sitzungen mit realer Akupunktur und manueller Nadelstimulation, 102 wurden an mindestens fünf von sieben Punkten genadelt, die nach der Lehre nicht als Akupunkturpunkte gelten („Schein-Akupunktur“), 108 wandten nur Cetirizin nach Maßgabe der Beschwerden an. Auch die Patienten der Gruppen mit realer und Scheinakupunktur konnten Cetirizin als zusätzliche Medikation verwenden, wenn es die Beschwerden erforderten.

Unter realer Akupunktur kam es bei einem Score der Lebensqualität und dem Verbrauch von Cetirizin nach 7-8 Wochen zu einer geringen, aber statistisch signifikanten Besserung bzw. Reduktion im Vergleich zu Scheinakupunktur und Cetirizin-Monotherapie, der sich in der 16. Woche nach Abschluss der Intervention wieder verflüchtigt hatte. Bei einem weiteren Follow-up im zweiten Jahr nach der Therapie war der Unterschied wieder in abgeschwächter Form nachweisbar. Die weiten Konfidenzintervalle weisen allerdings darauf hin, dass es offensichtlich große individuelle Unterschiede des Ansprechens gab, ein Umstand, der die Vorhersage im Einzelfall erschwert.

\footnotetext{
- B. Brinkhaus et al.

(Korres.: Benno Brinkhaus, Institut für Sozialmedizin, Epidemiologie und Gesundheitsökonomie, Charité, 10098 Berlin): Acupuncture in patients with seasonal allergic rhinitis. Ann. int. Medicine 2013; 158: 225-234.
}

\section{Kommentar}

Wieder eine Akupunkturstudie aus Deutschland, die einen etwas ratlos hinterlässt und die Frage aufwirft, ob es überhaupt möglich und sinnvoll ist, diese Therapieform mit den Methoden der wissenschaftlichen Medizin zu untersuchen. Vielleicht hatten die Forscher einfach nur Glück, dass wenigstens zu einem Zeitpunkt der Untersuchung ein statistisch signifikantes Ergebnis herauskam. Ob das für die Akzeptanz der Methode eine Rolle spielt, ist mehr als fraglich. Die Kostenträger haben die Segel angesichts der Liebe der Deutschen zur Akupunktur ohnehin gestrichen. Sie erstatten das Verfahren, obwohl die ebenfalls aus Deutschland stammenden Akupunkturstudien zur Arthrose gezeigt haben, dass es keine Rolle spielt, an welchen Punkte genadelt wird: Die Beschwerden bessern sich fast immer zumindest vorübergehend. Und wenn der Arzt wie ein Chinese aussieht, wirkt das Verfahren noch besser.

Über das Aussehen der nadelnden Ärzte haben die Autoren der vorliegenden Studie nichts mitgeteilt. Ob man tatsächlich, wie die Autoren vorschlagen, auch noch den Effekt in Abhängigkeit von den Erwartungen und a-priori-Einstellungen der Patienten untersuchen soll, erscheint überflüssig: Das Ergebnis kann man aufgrund der bisherigen Daten und der allgemeinen Erfahrungen als Arzt vorhersagen. Wer viel von Akupunktur hält, bei dem wirkt sie auch gut. Wer nichts davon hält, nimmt an so einer Studie erst gar nicht teil.

H. S. FÜESSL — 